\title{
Avaliação do nível de satisfação e expectativa dos pacientes em relação aos tratamentos restauradores estéticos
}

Evaluation of patients' level of satisfaction and expectations regarding aesthetic restorative treatments Evaluación del nivel de satisfacción y expectativa de los pacientes en relación a los tratamientos restauradores estéticos José Fábio Ferreira de SOUZA ${ }^{\mathbf{1}}$

Kauana da Silva ANDRADE ${ }^{2}$

Fábio Victor Dias SILVA2

Leonardo Henrique de Araújo CAVALCANTE ${ }^{3}$

${ }^{1}$ Cirurgião-Dentista pelo Centro Universitário de João Pessoa (UNIPÊ), 8053-000 João Pessoa - PB, Brasil

${ }^{2}$ Graduando(a) do Curso de Odontologia do Centro Universitário de João Pessoa (UNIPÊ), 8053-000 João Pessoa - PB, Brasil

${ }^{3}$ Professor Mestre do Curso de graduação em Odontologia do Centro Universitário de João Pessoa (UNIPÊ), 8053-000 João Pessoa - PB, Brasil

\section{Resumo}

Introdução: A satisfação refere-se ao acolhimento das necessidades e expectativas dos usuários, de forma que essas questões sejam atendidas e traduzidas em ofertas de ações e serviços de qualidade. Objetivo: Este estudo teve como objetivo avaliar o nível de satisfação e expectativa de pacientes em relação aos tratamentos restauradores estéticos. Metodologia: Estudo transversal realizado com 54 pacientes, atendidos na clínica odontológica de uma Instituição de Ensino Superior. Os pacientes responderam um questionário, baseado na Escala de Likert, sobre a expectativa e satisfação em relação aos tratamentos restauradores realizados. Os dados foram analisados de maneira descritiva e analítica por meio do teste de Fisher no software IBM SPSS Statistics, versão 20.0. Resultados: Dos 54 participantes, 66,7\% eram mulheres, com renda mensal de até um salário mínimo $(32,1 \%)$ e ensino superior completo $(43,4 \%)$. Além disso, $88,9 \%$ dos usuários estavam satisfeitos com a qualidade dos tratamentos restauradores e foi observada uma correlação entre a escolaridade e o grupo de dentes restaurados $(p=<0,05)$. Conclusão: Antes da realização dos procedimentos, os pacientes apresentavam altas expectativas, mas estavam satisfeitos quanto à qualidade do serviço odontológico ofertado. Os pacientes que possuíam o ensino fundamental apresentavam mais dentes anteriores com indicação para restauração, enquanto os pacientes com o ensino médio apresentavam indicação para procedimentos restauradores tanto nos dentes anteriores quanto nos dentes posteriores.

Descritores: Satisfação do Paciente; Percepção Visual; Odontologia.

\section{Abstract}

Introduction: Satisfaction refers to welcoming the needs and expectations of users, so that these issues are met and translated into offers of quality actions and services. Objective: This study aimed to assess the level of satisfaction and expectation of patients in relation to aesthetic restorative treatments. Methodology: Cross-sectional study carried out with 54 patients, seen at the dental clinic of a Higher Education Institution. The patients answered a questionnaire, based on the Likert Scale, about the expectation and satisfaction regarding the restorative treatments performed. The data were analyzed in a descriptive and analytical way using the Fisher test in the IBM SPSS Statistics software, version 20.0. Results: Of the 54 participants, $66.7 \%$ were women, with a monthly income of up to one minimum wage (32.1\%) and complete higher education (43.4\%). In addition, $88.9 \%$ of users were satisfied with the quality of the restorative treatments and a correlation was observed between schooling and the group of restored teeth $(p=<0.05)$. Conclusion: Before performing the procedures, patients had high expectations, but were satisfied with the quality of the dental service offered. Patients who had completed elementary school had more anterior teeth with indication for restoration, while patients with high school had indication for restorative procedures on both anterior and posterior teeth.

Descriptors: Patient Satisfaction; Visual Perception; Dentistry.

\section{Resumen}

Introducción: La satisfacción se refiere a acoger las necesidades y expectativas de los usuarios, para que estos temas sean atendidos y traducidos en ofertas de acciones y servicios de calidad. Objetivo: Este estudio tuvo como objetivo evaluar el nivel de satisfacción y expectativa de los pacientes en relación a los tratamientos restauradores estéticos. Metodología: Estudio transversal realizado con 54 pacientes, atendidos en la clínica odontológica de una Institución de Educación Superior. Los pacientes respondieron un cuestionario, basado en la Escala Likert, sobre la expectativa y satisfacción con respecto a los tratamientos restauradores realizados. Los datos se analizaron de forma descriptiva y analítica mediante la prueba de Fisher en el software IBM SPSS Statistics, versión 20.0. Resultados: De los 54 participantes, el 66,7\% eran mujeres, con un ingreso mensual de hasta un salario mínimo (32,1\%) y estudios superiores completos (43,4\%). Además, el $88,9 \%$ de los usuarios se mostró satisfecho con la calidad de los tratamientos restauradores y se observó una correlación entre la educación y el grupo de dientes restaurados $(\mathrm{p}=<0,05)$. Conclusión: Antes de realizar los procedimientos, los pacientes tenían altas expectativas, pero estaban satisfechos con la calidad del servicio odontológico ofrecido. Los pacientes que habían completado la escuela primaria tenían más dientes anteriores con indicación de restauración, mientras que los pacientes con estudios secundarios tenían indicación para procedimientos de restauración tanto en dientes anteriores como en dientes posteriores.

Descriptores: Satisfacción del Paciente; Percepción Visual; Odontología.

INTRODUÇÃO

A satisfação refere-se ao acolhimento das necessidades e expectativas dos usuários em relação aos serviços, de forma que essas questões sejam atendidas e traduzidas em ofertas de ações e serviços de qualidade ${ }^{1}$. Atualmente, além de uma condição bucal satisfatória, os pacientes têm buscado uma aparência harmônica, objetivando 0 estabelecimento do equilíbrio estético e funcional dos dentes, assim como a sua satisfação estética diante da sociedade ${ }^{2}$.
As clínicas odontológicas das Instituições de Ensino Superior (IESs) oferecem atendimento realizado por acadêmicos, sob a supervisão de profissionais experientes. Essas instituições de ensino atendem uma demanda considerável da população devido ao custo e efetividade dos tratamentos ${ }^{3,4}$. Com isso, é preciso mensurar a qualidade do serviço disponibilizado, visto que os pacientes estão cada vez mais exigentes, pois têm buscado padrões estéticos que proporcionem segurança 
e autoestima, para tanto, os pacientes buscam um sorriso considerado harmônico, cujos dentes sejam brancos e simétricos ${ }^{5}$.

A satisfação do usuário é uma avaliação pessoal dos cuidados e serviços de saúde que são ofertados e de extrema relevância para mensurar o padrão de serviço disponibilizado. Com isso, a satisfação do paciente permite avaliar as lacunas no tratamento e, a partir disso, a busca por mecanismos que visem a superação das limitações encontradas ${ }^{5}$.

Diante do exposto, o presente trabalho teve como objetivo avaliar a satisfação e expectativa dos pacientes submetidos a tratamentos restauradores estéticos realizados em uma Instituição de Ensino Superior (IES), na Paraíba. Dessa forma, verificou-se o nível de expectativa antes da realização dos procedimentos, assim como a satisfação com o tratamento finalizado.

MATERIAL E MÉTODO

Trata-se de um estudo transversal com abordagem descritiva e exploratória, realizado em uma Instituição de Ensino Superior (IES), localizada na cidade de João Pessoa - PB, Brasil. $O$ universo foi constituído pelos usuários de demanda espontânea dos serviços ofertados pela clínica odontológica da IES, sendo uma amostra do tipo não probabilística, selecionando, por conveniência, 54 pacientes.

Foram incluídos pacientes maiores de dezoito anos, atendidos na clínica da IES, submetidos a tratamentos restauradores estéticos com resina composta, resina flow, resina acrílica e cerômero, assim como pacientes com coroas e facetas definitivas em resina composta. Enquanto os critérios de exclusão foram os pacientes com próteses totais, superiores e inferiores, restaurações provisórias, além dos pacientes com clareamento dental e os portadores de deficiências visuais e intelectuais.

O instrumento de coleta de dados foi um questionário e as variáveis analisadas contemplavam a caracterização da amostra de acordo com os dados sociodemográficos, além de questões específicas sobre a expectativa e satisfação com os tratamentos restauradores estéticos realizados pelos acadêmicos. Para tanto, o questionário foi baseado na Escala Analógica Visual de Likert, onde o participante avaliou de 1 a 4 pontos, a expectativa e satisfação com o tratamento realizado, sendo considerado 1 como pouco insatisfeito e 4 como satisfeito.

Todos os participantes assinaram 0 Termo de Consentimento Livre e Esclarecido (TCLE) e a coleta de dados só teve início após a aprovação pelo Comitê de Ética em Pesquisa do Centro Universitário de João Pessoa (UNIPÊ), com CAAE 12020118.6.0000.5176.

Os dados foram analisados de maneira descritiva com frequências absolutas e percentuais para as variáveis categóricas, assim como medidas de tendência central e de variabilidade para as variáveis quantitativas por meio software IBM SPSS Statistics, versão 20.0. Em seguida, realizou-se o teste de Fisher, com nível de significância estatística de 5\%, para determinar associação entre escolaridade, grupo de dentes restaurados e o nível de expectativa e satisfação com o tratamento restaurador.

\section{RESULTADOS}

Dos 54 participantes, $66,7 \%$ eram do sexo feminino, com renda mensal de até um salário mínimo $(32,1 \%)$ e declararam possuir o ensino superior completo $(43,4 \%)$, conforme a Tabela 1.

Tabela 1. Caracterização dos pacientes de acordo com os dados sociodemográficos (João Pessoa - PB, Brasil - 2019)

\begin{tabular}{l|l|l}
\hline Variáveis & $\mathbf{N}$ & $\mathbf{\%}$ \\
\hline Sexo & & \\
\hline Masculino & 18 & $33,3 \%$ \\
\hline Feminino & 36 & $66,7 \%$ \\
\hline Renda econômica & & \\
\hline Nenhuma & 12 & $22,6 \%$ \\
\hline Até 1 salário mínimo & 17 & $32,1 \%$ \\
\hline De 1 a 3 salários mínimos & 16 & $30,2 \%$ \\
\hline De 3 a 6 salários mínimos & 7 & $13,2 \%$ \\
\hline Mais de 6 salários mínimos & 1 & $1,9 \%$ \\
\hline Escolaridade & & \\
\hline Ensino fundamental completo & 4 & $7,5 \%$ \\
\hline Ensino fundamental incompleto & 8 & $15,1 \%$ \\
\hline Ensino médio completo & 11 & $20,8 \%$ \\
\hline Ensino médio incompleto & 3 & $5,7 \%$ \\
\hline Ensino superior completo & 23 & $43,4 \%$ \\
\hline Ensino superior incompleto & 4 & $7,5 \%$ \\
\hline Total & 54 & $100 \%$ \\
\hline Fonte: Dados da pesquisa (2019). & \multicolumn{2}{|l}{}
\end{tabular}

De acordo com a Tabela 2, verificou-se que a maioria dos entrevistados $(98,1 \%)$ não determinou nenhum tipo de material restaurador para a realização do procedimento, sendo a escolha feita pelo acadêmico. Os dentes mais comumente restaurados foram os dentes posteriores $(48,1 \%)$, sendo $56,1 \%$ os molares.

O nível de expectativa, antes da realização do tratamento, mostrou-se alto, correspondendo a $43,4 \%$, enquanto $88,9 \%$ dos pacientes relataram estar satisfeitos com 0 procedimento realizado. Além disso, 94,4\% dos entrevistados retornariam para novos atendimentos na IES, sendo um valor superior ao nível de satisfação (Tabela 2).

A Tabela 3 descreve a associação significativa entre escolaridade e grupo de dentes restaurados $(p=0,014)$. Os pacientes com ensino fundamental apresentavam mais dentes anteriores com indicação para restauração $(n=6 ; 50 \%)$, à medida que os pacientes com o ensino médio, frequentemente, apresentavam tanto dentes anteriores quanto posteriores com indicação para procedimentos 
restauradores ( $n=6 ; 42,9 \%)$.

Tabela 2. Distribuição dos participantes de acordo com a expectativa e satisfação com o tratamento restaurador estético (João Pessoa - PB, Brasil - 2019)

\begin{tabular}{l|l|l}
\hline Variáaeis & $\mathrm{N}$ & $\%$ \\
\hline Satisfacão com o tratamento restaurador & & \\
\hline Pouco insatisfeito & 2 & $3,7 \%$ \\
\hline Razoavelmente satisfeito & 2 & $3,7 \%$ \\
\hline Pouco satisfeito & 2 & $3,7 \%$ \\
\hline Satisfeito & 48 & $88,9 \%$ \\
\hline Exigência por algum material restaurador & & \\
\hline Sim, resina composta & 1 & $1,9 \%$ \\
\hline Não & 53 & $98,1 \%$ \\
\hline Grupo de dentes restaurados & & \\
\hline Anteriores & 13 & $24,1 \%$ \\
\hline Posteriores & 26 & $48,1 \%$ \\
\hline Ambos & 15 & $27,8 \%$ \\
\hline Dentes anteriores restaurados & & \\
\hline Incisivos & 15 & $53,6 \%$ \\
\hline Caninos & 10 & $35,7 \%$ \\
\hline Ambos & 3 & $10,7 \%$ \\
\hline Dentes posteriores restaurados & & \\
\hline Pré-molares & 10 & $24,4 \%$ \\
\hline Molares & 23 & $56,1 \%$ \\
\hline Ambos & 8 & $19,5 \%$ \\
\hline Nível de expectativa antes do tratamento & & \\
\hline Nenhum & 3 & $5,7 \%$ \\
\hline Baixo & 4 & $7,5 \%$ \\
\hline Razoável & 5 & $9,4 \%$ \\
\hline Alto & 23 & $43,4 \%$ \\
\hline Muito alto & 18 & $34 \%$ \\
\hline Satisfacãao com o tratamento finalizado & & \\
\hline Pouco insatisfeito & 1 & $1,9 \%$ \\
\hline Razoavelmente satisfeito & 2 & $3,7 \%$ \\
\hline Pouco satisfeito & 3 & $5,6 \%$ \\
\hline Satisffito & 48 & $88,9 \%$ \\
\hline Se retornaria a clínica da IES para novos atendimentos & & \\
\hline Sim & 51 & $94,4 \%$ \\
\hline Não & 3 & $5,6 \%$ \\
\hline Conhecimento que o procedimento seria realizado por acadêmicos & & \\
\hline Sim & 48 & $88,9 \%$ \\
\hline Não & 6 & $11,1 \%$ \\
\hline Total & 54 & $100 \%$ \\
\hline Fonte: Dados da pesquisa (2019). & & \\
\hline
\end{tabular}

Tabela 3. Associação entre escolaridade e nível de expectativa e satisfação com o tratamento restaurador (João Pessoa - PB, Brasil - 2019)

\begin{tabular}{|c|c|c|c|c|c|c|c|c|c|}
\hline \multirow{3}{*}{ Variável } & \multicolumn{6}{|c|}{ Escolaridade } & & & \multirow[b]{2}{*}{$\begin{array}{l}\text { Valor } \\
\text { de p* }\end{array}$} \\
\hline & \multicolumn{2}{|c|}{\begin{tabular}{|c|} 
Ensino \\
fundamental
\end{tabular}} & \multicolumn{2}{|c|}{$\begin{array}{c}\text { Ensino } \\
\text { médio }\end{array}$} & \multicolumn{2}{|c|}{$\begin{array}{l}\text { Ensino } \\
\text { superior }\end{array}$} & \multicolumn{2}{|c|}{ Total } & \\
\hline & $\mathbf{N}$ & $\%$ & $\mathbf{N}$ & $\%$ & $\mathbf{N}$ & $\%$ & $\mathbf{N}$ & $\%$ & \\
\hline \multicolumn{9}{|c|}{ Satisfação com o tratamento restaurador } & 0,123 \\
\hline $\begin{array}{l}\text { Pouco } \\
\text { insatisfeito }\end{array}$ & 1 & $8,3 \%$ & o & - & 1 & $3,7 \%$ & 2 & $3,8 \%$ & \\
\hline $\begin{array}{l}\text { Razoavelment } \\
\text { e satisfeito }\end{array}$ & 0 & - & 0 & - & 2 & $7,4 \%$ & 2 & $3,8 \%$ & \\
\hline $\begin{array}{l}\text { Pouco } \\
\text { satisfeito }\end{array}$ & 2 & $16,7 \%$ & o & - & o & - & 2 & $3,8 \%$ & \\
\hline \multirow{2}{*}{\multicolumn{9}{|c|}{ algum material restaurador }} & \\
\hline & & & & & & & & & 1,000 \\
\hline Sim & 0 & & 0 & & 1 & $3,7 \%$ & 1 & $1,9 \%$ & \\
\hline & 12 & $100 \%$ & 14 & $100 \%$ & 26 & $96,3 \%$ & 52 & $98,1 \%$ & \\
\hline \multicolumn{9}{|c|}{ Grupo de dentes restaurados } & 0,014 \\
\hline Anteriores & 6 & $50 \%$ & 4 & $28,6 \%$ & 3 & $11,1 \%$ & 13 & $24,5 \%$ & \\
\hline Posteriores & 3 & $25 \%$ & 4 & $28,6 \%$ & 1 & $70,4 \%$ & 26 & $49,1 \%$ & \\
\hline Ambos & 3 & $25 \%$ & 6 & $42,9 \%$ & 5 & $18,5 \%$ & 14 & $26,4 \%$ & \\
\hline \multicolumn{9}{|c|}{ Dentes anteriores restaurados } & 0,72 \\
\hline Inci: & 5 & $55,6^{6}$ & 5 & $50 \%$ & 4 & $50 \%$ & 14 & $51,9 \%$ & \\
\hline Canin & 2 & $22,2 \%$ & 4 & $40 \%$ & 4 & $50 \%$ & 10 & $37 \%$ & \\
\hline Ambos & 2 & $22,2 \%$ & 1 & $10 \%$ & $\mathrm{o}$ & 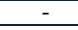 & 3 & $11,1 \%$ & \\
\hline \multicolumn{9}{|c|}{ Dentes posteriores restaurados } & 0,098 \\
\hline Pré-molares & 1 & $16,7 \%$ & 2 & $20 \%$ & 7 & $31,8 \%$ & 10 & $26,3 \%$ & \\
\hline Molares & 5 & $83,3 \%$ & 4 & $40 \%$ & 1 & $63,6 \%$ & 23 & $60,5 \%$ & \\
\hline Ambos & 0 & & 4 & $40 \%$ & 1 & $4,5 \%$ & 5 & $13,2 \%$ & \\
\hline \multicolumn{9}{|c|}{ Nível de expectativa antes do tratamento } & 0,13 \\
\hline Nenhum & 0 & & 2 & $14,3 \%$ & 1 & $3,7 \%$ & 3 & $5,8 \%$ & \\
\hline Baixo & 2 & $18,2 \%$ & 1 & $7,1 \%$ & 1 & $3,7 \%$ & 4 & $7,7 \%$ & \\
\hline Razoável & 1 & $9,1 \%$ & $\mathrm{O}$ & & 4 & $14,8 \%$ & 5 & $9,6 \%$ & \\
\hline Alto & 3 & 27,3 & 4 & 28,6 & 15 & $\begin{array}{c}55,6 \\
0\end{array}$ & 2 & 42,3 & \\
\hline Muito alto & 5 & $45.5 \%$ & 7 & $50 \%$ & 6 & $22,2 \%$ & 18 & $34,6 \%$ & \\
\hline \multicolumn{9}{|c|}{ Satisfação com o tratamento finalizado } & 0,205 \\
\hline $\begin{array}{l}\text { Pouco } \\
\text { insatisfe }\end{array}$ & 1 & $8,3 \%$ & o & - & $\mathrm{o}$ & - & 1 & $1,9 \%$ & \\
\hline $\begin{array}{l}\text { Razoavelment } \\
\text { e satisfeito }\end{array}$ & o & - & 1 & $7,1 \%$ & 1 & $3,7 \%$ & 2 & $3,8 \%$ & \\
\hline $\begin{array}{l}\text { Pouco } \\
\text { satisfei }\end{array}$ & 2 & $16,7 \%$ & o & - & 1 & $3,7 \%$ & 3 & $5,7 \%$ & \\
\hline Satisfeito & & $75 \%$ & 13 & & 25 & $92,6 \%$ & 47 & $88,7 \%$ & \\
\hline \multicolumn{9}{|c|}{ Se retornaria a clínica da IES para novos atendimentos } & 0,597 \\
\hline Sim & 11 & $91,7 \%$ & 14 & $100 \%$ & 25 & $92,6 \%$ & 50 & $94,3 \%$ & \\
\hline Não & 1 & $8,3 \%$ & $\mathrm{O}$ & 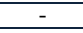 & 2 & $7,4 \%$ & 3 & $5,7 \%$ & \\
\hline
\end{tabular}

\section{DISCUSSÃO}

O cuidado centrado no paciente é um aspecto fundamental na prática odontológica, no entanto, uma revisão sistemática demonstra que as evidências em relação à compreensão deste cuidado são limitadas. Associado a isso, ressalta-se a importância de pesquisas realizadas a partir da perspectiva do paciente como um indicador de qualidade do serviço ofertado ${ }^{6}$.

Este estudo avaliou a satisfação e expectativa dos pacientes quanto aos tratamentos restauradores estéticos, constituindo-se um dos poucos estudos desenvolvidos no Brasil com 0 objetivo de investigar a associação dos níveis de expectativa e satisfação com fatores sociodemográficos.

A prevalência de pacientes do sexo feminino $(66,7 \%)$ e com renda familiar até 1 salário mínimo (32,1\%) observada neste estudo está em consonância com um estudo transversal realizado com 8943 brasileiros, onde $62,9 \%$ dos participantes eram mulheres e $66 \%$ apresentavam uma renda igual ou inferior a $\mathrm{R} \$ 1500,00^{7}$.

Em relação a satisfação com os tratamentos restauradores estéticos, verificouse que a maioria dos participantes (88,9\%) estavam satisfeitos. Esse achado corrobora os níveis de satisfação observados em estudos anteriores, onde $(78 \%)^{1}$ e $(89 \%)^{8}$ dos participantes afirmaram estar satisfeitos com o tratamento odontológico oferecido. Entretanto, ressalta-se que nos referidos estudos, a satisfação dos usuários esteve mais relacionada à maneira como eles foram tratados e com a resolutividade do problema, do que com os aspectos técnicos da assistência propriamente dita.

A elevada satisfação com o serviço, observada nesta pesquisa, está de acordo com os resultados sobre a satisfação dos usuários com os serviços odontológicos no Brasil, tanto públicos quanto privados, em diferentes grupos etários ${ }^{9}$.

Constatou-se que $98,1 \%$ dos entrevistados não determinou o tipo de material restaurador, sendo a escolha realizada pelo acadêmico, supervisionado por um profissional experiente. Em contrapartida, um estudo evidenciou que os pacientes preferem o uso da resina composta para realização dos procedimentos restauradores e que essa exigência tem aumentando ao decorrer dos anos $^{10}$.

Os elementos dentários mais comumente restaurados foram os posteriores 
$(48,1 \%)$, sendo os molares, os dentes mais restaurados $(56,1 \%)$. A frequência de restaurações nos molares pode ser justificada pelo não só pelo contato frequente com 0 alimento, como também pelas maiores cargas diretas que estes elementos dentários recebem, o que aumentaria a probabilidade de falhas ${ }^{11}$.

O nível de expectativa, antes da realização do tratamento, foi considerado alto $(43,4 \%)$, enquanto a satisfação após o tratamento correspondeu a $88,9 \%$ dos participantes. Associado a isso, $94,4 \%$ dos entrevistados retornariam à clínica da IES para novos os atendimentos. $O$ fato do tratamento não ter custo para o paciente pode interferir nos níveis de satisfação com o serviço. Considerando que a IES oferta atendimentos de forma gratuita, pode-se ter, nesta constatação, o alto nível de satisfação e, ao mesmo tempo, o alto índice de pacientes que afirmaram retornar para outros atendimentos, inclusive os que não se declararam totalmente satisfeitos ${ }^{12}$.

Além disso, 88,9\% dos participantes sabiam que seriam atendidos por acadêmicos. Neste contexto, um estudo realizado em uma clínica escola da Paraíba observou que a maioria dos pacientes $(56,2 \%)$ se sentiam seguros e satisfeitos ao serem atendidos por um acadêmico, independentemente do mesmo estar sob a supervisão de um professor ${ }^{13}$. Esse dado está associado a atenção que os acadêmicos têm com os pacientes, criando uma situação de cordialidade que fortalece a confiança e a credibilidade no atendimento.

O grau de satisfação do usuário pode ser mensurado a partir de fatores como o nível de escolaridade, a autopercepção, a condição socioeconômica e a qualidade de vida, assim como a qualidade do tratamento realizado ${ }^{14}$. Estudos evidenciaram que pacientes com menor escolaridade e com renda inferior a 2 salários mínimos tinham experiências de cárie, de forma mais severa, e apresentavam maior predisposição as lesões de cárie e perdas dentárias ${ }^{15-18}$.

Os resultados descritos indicam a necessidade de estudos que avaliem a percepção dos pacientes sobre a expectativa e satisfação dos serviços odontológicos em IESs, analisando a conduta dos acadêmicos, especialmente em relação aos procedimentos estéticos, que têm impacto na autoestima e integração social dos pacientes. Assim como, a busca por subsídios que forneçam a implementação de medidas preventivas a partir da problematização do cenário social, buscando minimizar a necessidade de intervenções invasivas, o que poderia certamente garantir uma melhor preparação dos acadêmicos para 0 mercado de trabalho ${ }^{19}$.

Dessa forma, os resultados do presente estudo podem orientar as instituições no planejamento e execução das estratégias de ensino, visando atendimentos fundamentados nas necessidades e desejos dos pacientes. Assim como, favorecer a formação de profissionais mais humanizados e que ofereçam um serviço de qualidade.

CONCLUSÃO

Os pacientes apresentavam altas expectativas antes da realização dos procedimentos, mas estavam satisfeitos quanto à qualidade do serviço odontológico ofertado. Em relação à satisfação e expectativa dos pacientes, foi observada uma associação significativa ao correlacionar 0 grau de escolaridade com 0 grupo de dentes restaurados. Dessa forma, os pacientes que possuíam o ensino fundamental apresentavam mais dentes anteriores com indicação para restauração, enquanto os pacientes com o ensino médio apresentavam indicação para procedimentos restauradores nos dentes anteriores e posteriores.

REFERÊNCIAS

1. Magalhães BM, Oliveira RS, Goes PSA, Figueiredo N. Avaliação da qualidade dos serviços prestados pelos Centros de Especialidades Odontológicas: visão dos usuários. Cad Saúde Colet. 2015;23(1):76-85.

2. Santos DT, Dias KR, Cervantes $H$, Santos MPA. Amálgama dental e seu papel na Odontologia atual. Rev Bras Odonto. 2016; 1(73):64-68.

3. Habib SR, Ramalingam S, Al Beladi A, Al Habib A. Patient's satisfaction with the dental care provided by dental students. J Ayub Med Coll Abbottabad. 2014;26(3):353-56.

4. Al-Hussyeen AJ. Factors affecting utilization of dental health services and satisfaction among adolescent females in Riyadh City. Saudi Dent J. 2010;22(1):19-25.

5. Becerra SG, Becerra MN, Jiménez AM, Medina PVM, Tamayo LLC, Gómez SL. Some factors actors associated to cosmetic dentistry: a new approach. Rev Fac Odontol Univ Antioq. 2015;26(2): 271-91.

6. Mills I, Frost J, Cooper C, Moles DR, Kay E. Patient-centred care in general dental practice a systematic review of the literature. BMC Oral Health. 2014;14 (64):1-13.

7. Carreiro DL, Oliveira RFR, Coutinho WLM, Martins AMEBL, Haikal DS. Avaliação da satisfação com a assistência odontológica na perspectiva de usuários brasileiros adultos: análise multinível. Ciên Saúde Colet. 2018; 23(12):4339-49. 
8. Sousa TL, Araújo TC, Souza CN. Avaliação da Satisfação dos pacientes Atendidos da Clínica escola de Ensino Superior. Rev Interfaces. 2015;3(8):1-6.

9. Macarevich A, Pilotto LM, Hildgert JB, Celeste RK. User satisfaction with public and private dental services for different age groups in Brazil. Cad Saúde Pública. 2018;34(2):1-10.

10. Sarac D, Sarac YS, Kulunk S, Ural C, Kulunk T. The effect of polishing techniques on the surface roughness and color change of composite resins. J Prosthet Dent. 2006; 96(1):33-40.

11. Veras BML, Menezes GPS, Gomes Filho VV, Silva CHV. Comportamento clínico de resinas compostas em dentes posteriores - revisão sistematizada da literatura. Odontol Clín-Cient. 2015;14(3):689-94.

12. Martins AMEBL, Jardim LA, Souza JGS, Rodrigues CAQ, Ferreira RC, Pordeus IA. A avaliação negativa dos serviços odontológicos entre idosos brasileiros está associada ao tipo de serviço utilizado? Rev Bras Epidemiol. 2014; 17(1):71-90.

13. Mota LQ, Farias DBLM, Santos TA. Humanização no atendimento odontológico: acolhimento da subjetividade dos pacientes atendidos por alunos de graduação em Odontologia. Arq Odontol. 2012;48(3):151-58.

14. Čelebić A, Knezović-Zlatarić D, Papić M, Carek V, Baučić I, Stipetić J. Factors related to patient satisfaction with complete denture therapy. J Gerontol a Biol Sci Med Sci. 2003;58(10): 948-53.

15. Batista MJ, Lawrence HP, Sousa MLR. Classificação das perdas dentárias: fatores associados a uma nova medida em uma população de adultos. Ciên Saúde Colet. 2015; 20(9):2825-35.

16. Carreiro DL, Souza JGS, Coutinho WLM, Ferreira RC, Ferreira EF, Martins AMEBL. Uso de serviços odontológicos de forma regular na população de Montes Claros, MG, Brasil. Ciênc Saúde Colet. 2017; 22(12):4135-50.

17. Lima CV, Souza JGS, Oliveira BEC, Noronha MS, Pereira AC, Probst LF. Falta de dentição funcional influencia na autopercepção da necessidade de tratamento em adultos: estudo de base populacional no Brasil. Cad Saúde Colet. 2018;26(1):63-9.

18. Schwendicke F, Dörfer CE, Schlattmann P, Foster Page L, Thomson WM, Paris $S$. Socioeconomic inequality and caries: a systematic review and meta-analysis. J Dent Res. 2015;94(1):10-8.

19. Oliveira JJM, Almeida NN, Farias MF, Andrade KS, Silva FVD, Lima Júnior MAV. Inclusão dos temas de gestão nos currículos dos cursos de graduação em Odontologia no Brasil. Arch Health Invest. 2021;10(1):82-6.

\section{CONFLITO DE INTERESSES}

Os autores declaram não haver conflitos de interesse

\section{AUTOR PARA CORRESPONDÊNCIA}

\author{
Kauana da Silva Andrade \\ BR $230-\mathrm{Km} 22$, \\ 58053-000 João Pessoa - PB, Brasil \\ E-mail: kauanaandrade12@gmail.com
}

Submetido em 11/04/2021

Aceito em 07/07/2021 